\title{
Research of Water Hammer of Air Conditioning Chilled Water System Based on Flowmaster
}

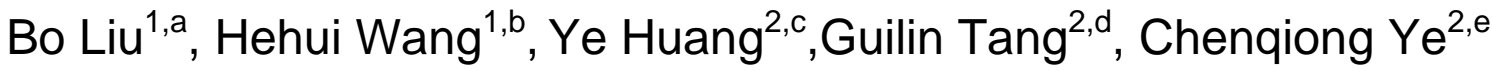 \\ ${ }^{1}$ School of Mechanical and Power Engineering, East China University of Science and \\ Technology, Shanghai, 200237, China \\ ${ }^{2}$ General Construction Company of CCTEB Group Co.,Ltd, Wuhan, 430064,China;

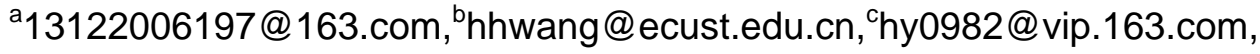 \\ dtgl19771977@163.com, èycq59642502@163.com
}

Keywords: water hammer, Flowmaster, pressure fluctuations.

\begin{abstract}
Water hammer phenomenon often occurs in the high-rise buildings air conditioning chilled water system, and cause enormous damage. With the help of Flowmaster platform, network model was built to simulate water hammer with different pipeline models, and the simulation results show that the water hammer is significantly inhibited after using modified models.
\end{abstract}

\section{Introduction}

Water hammer phenomenon is often caused by sudden changes in velocity during the long-distance transmission, high-lift, opening and closing the valve or the pump in piping system, which in turn leads to the pressure increasing or decreasing and kept propagating and reflecting back and forth along the pipeline in the form of. The main physical reason for the occurrence of water hammer is the liquid inertia and compressibility [1].

Method of characteristics is the most commonly used numerical method to solve potential water hammer problems, which can make ordinary differential equations to be converted into partial differential equations, and finite difference equations can be obtained by this method. This method has effectively and successfully applied to water hammer analysis in complex piping systems and boundary conditions for its easy computer programming, computational accuracy and efficiency advantages [2].

According to the elastic water hammer theory, the movement equation and the continuum equation are expressed as follows:

$$
\begin{aligned}
& \frac{\partial H}{\partial x}+\frac{1}{g} \frac{\partial V}{\partial t}+\frac{V}{g} \frac{\partial V}{\partial x}+\frac{f}{D} \frac{V|V|}{2 g}=0 \\
& \frac{\partial H}{\partial t}+V\left(\frac{\partial H}{\partial x}+\sin \theta\right)+\frac{\alpha^{2}}{g} \frac{\partial V}{\partial x}=0
\end{aligned}
$$

Where $H$ is the water head of a point, $f$ is pipe friction coefficient, $V$ is inner tube velocity $(\mathrm{m} / \mathrm{s}), \square$ is the angle between the piping and the horizontal plane, $a$ is propagation velocity of water hammer wave, $x$ is position coordinates, $t$ is propagation time of water hammer wave, $D$ is pipe diameter.

Using characteristic method to solve water hammer problem, first it is necessary that transient partial differential equation which can not be solved directly should be transformed into a special total differential equation, which is called the characteristic line equation. Then, resulting in an approximate algebraic integral type or finite difference equation by the integral of differential equations, the finite difference equation is shown as follows:

$$
C^{+}\left\{\begin{array}{l}
\frac{d H}{d t}+\frac{\alpha}{g} \frac{d V}{d t}+V_{\sin \theta}+\frac{\alpha f}{2 g D} V|V|=0 \\
\frac{d x}{d t}=V+\alpha
\end{array}\right.
$$




$$
C^{-}\left\{\begin{array}{l}
\frac{d H}{d t}-\frac{\alpha}{g} \frac{d V}{d t}+V_{S i n} \theta-\frac{\alpha f}{2 g D} V|V|=0 \\
\frac{d x}{d t}=V-\alpha
\end{array}\right.
$$

Where $\mathrm{C}^{+}$denotes a positive characteristic equation and $\mathrm{C}^{-}$indicates a negative characteristic line equation.

\section{Model}

An air conditioning chilled water system of a high-rise building includes three water pumps, six butterfly valves, three check valves, a main pipeline with a diameter of DN700mm, three branching pipelines with different diameters of DN350mm, DN500mm and DN450mm that respectively provide cold water coming from the main pipeline to the thirty-one floor $(160 \mathrm{~m})$, the fifty-five floor $(280 \mathrm{~m})$ and the seventy-nine floor $(400 \mathrm{~m})$.

The main function of fluid analysis software FLOWMASTER is to accurately analyze pressure, flow, temperature and velocity of each part of piping system, which can quickly obtain the above mentioned parameters of the numerical simulation from the established chilled water system and carries on the analysis comparison with the design values. This paper introduced the application of FLOWMASTER in the hydraulic balance of air conditioning chilled water system [2].

There are dedicated calculation models for water pumps, check valves, butterfly valves, pipelines, etc. in FLOWMASTER. Usually, it has strong generality and versatility for calculation models of tank and piping relative to pumps and valves, so it is necessary to obtain the actual value of the specific parameter from equipment manufacturers in order to simulate real hydraulic characteristics.

In this paper, the simulation parameters of water pumps are real provided by manufacturer, but the curves of valve opening degree is failed to provided, which follows the default setting of FLOWMASTER, while the action time of the valve were defined according to the work scene [3].

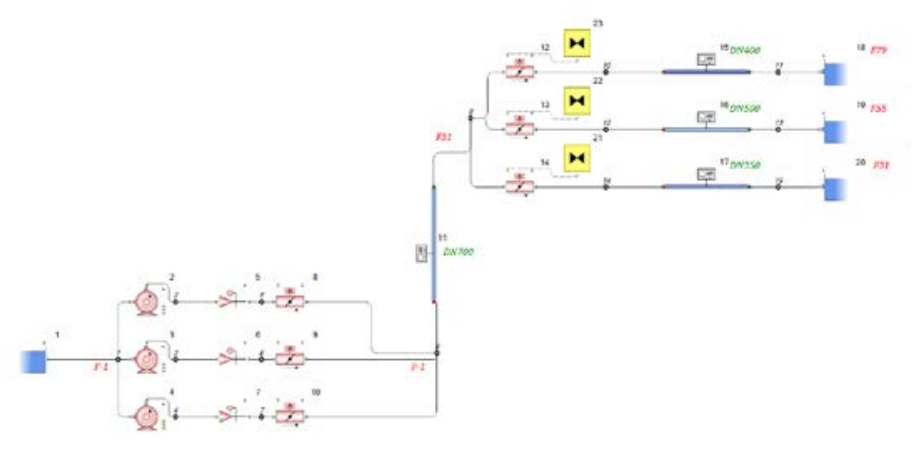

Fig.1 Model network diagram

Fig. 1 is the model network diagram of the air conditioning chilled water system, which includes a total of 26 elements and 15 nodes, and elements 1、18、19、20 are water tank, elements 2、3、4 are pumps, elements 5、6、 7 are check valves, elements $8 、 9 、 10 、 12 、 13 、 14$ are butterfly valves, elements 21、22、23 are valve control elements, element 11 is main pipeline of DN700mm, elements 15、16、17 are branch pipes.

\section{Analysis}

By definition of horizontal high of different nodes, it can be implemented that cold water is transported from low-rise pumping house to high-rise water tank. The initiation of the setups are that all pumps are working properly and check valves fully open, and butterfly valves operate on the defined action time of butterfly valve opening degree. Assuming that three butterfly valves quickly 
completely closed within 4 seconds, whether the downstream piping would produce water hammer phenomenon, the simulation result is showing in Fig.2.

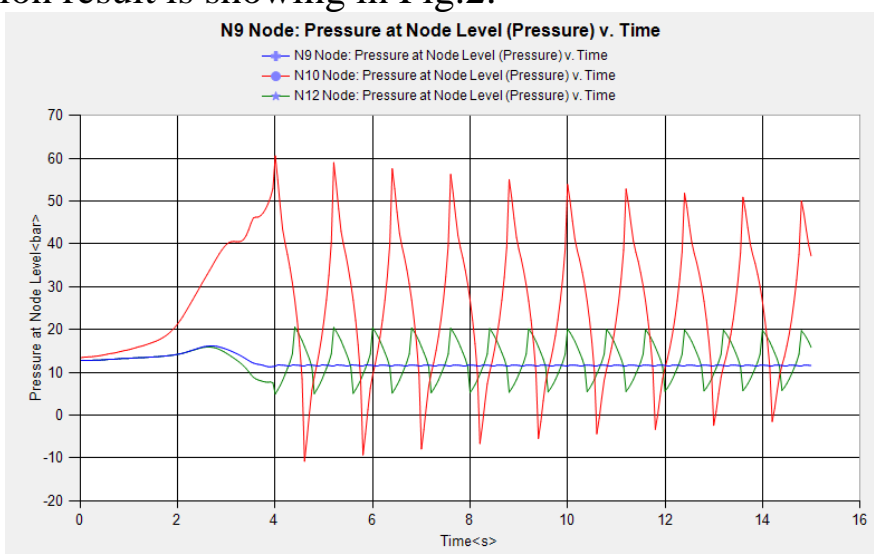

Fig. 2 The variation of pressure over time for nodes 9、10、12

Fig. 2 compares the pressure fluctuation related to the time of nodes $9 、 10 、 12$, and it can be conclude that the maximum water hammer wave occurred in node 10 within 4 seconds, and the node pressure from the initial pressure $1.4 \mathrm{MPa}$ rapidly increased to the peak pressure $6 \mathrm{MPa}$. Therefore, it can be determined that the operation of butterfly valves quickly completely closed within 4 seconds would lead to a violent water hammer.

\section{Modification}

The harm of water hammer phenomenon has put forward a severe test to the whole pipeline system. For this phenomenon, this paper puts forward two kinds of modifications to the model in order to decrease the node pressure fluctuation: (a) it could increase the valve closing time, especially adopting slowly closing pattern; (b) it can adopt regulated water tank connected with valves.

Set 1: Half opening degree of three butterfly valves is fast closed within 2 seconds, and the surplus opening degree is completed within 38 seconds.

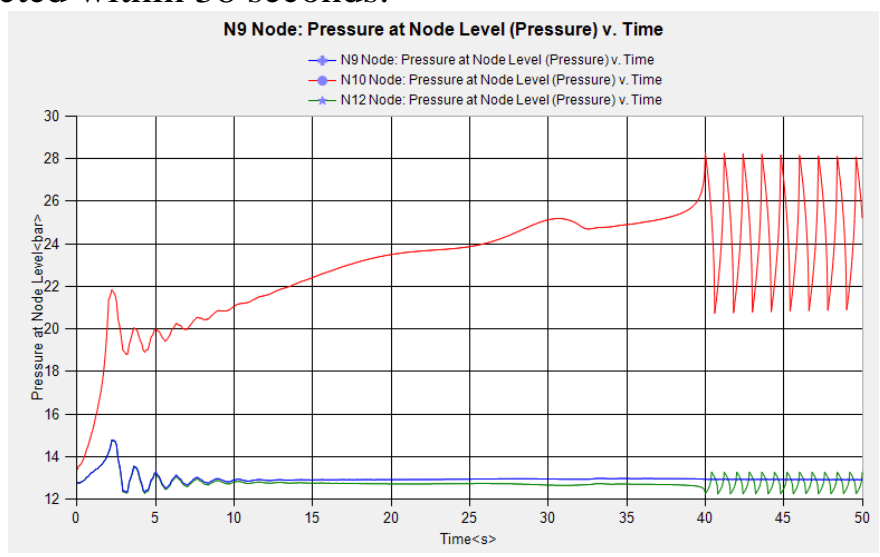

Fig.3 The variation of pressure over time for nodes 9、10、12

Fig. 3 shows that the pressure fluctuation of node 10 has obviously decreased within 4 seconds, which node pressure from the initial pressure 1.4 MPa slowly increased to the peak pressure 2.8 MPa after correction, and the pressure fluctuation of node 12 also has a certain amount of decrease. Therefore, it can be determined that the water hammer is significantly inhibited when adopting a slow closing valve operation.

Set 2: Add regulated water tank after the node 10. 


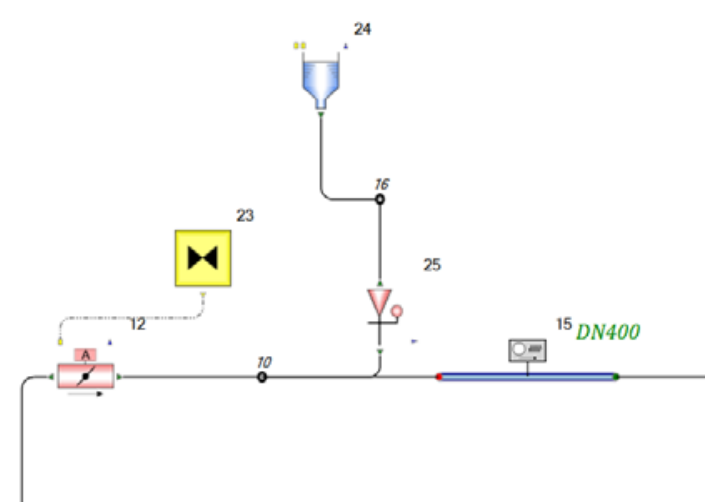

Fig.4 Add regulated water tank

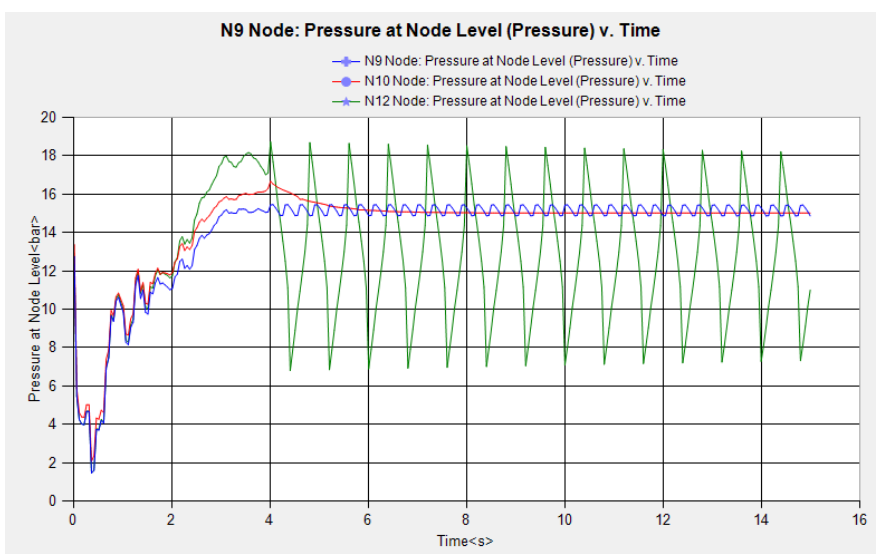

Fig.5 The variation of pressure over time for node 9、10、12

Fig. 4 shows the modified model added regulated water tank. Fig. 5 shows that the pressure of node 10 from the initial pressure 1.4 MPa increased to the peak pressure $1.9 \mathrm{MPa}$ within 4 seconds, and downstream pipeline generated a low degree of pressure fluctuation after butterfly valves completely closed. Therefore, it can be determined that the water hammer is significantly inhibited after adding a regulated water tank.

\section{Conclusion}

When there are not using disincentive measures and three butterfly valves quickly completely closed within 4 seconds, it would lead to a significant water hammer effect of branch pipeline, and the node peak pressure can reach $6 \mathrm{MPa}$, which means a huge potential security problem. After using modified models, simulation results show that the two modified models are more conducive to the stability of the pipeline and the water hammer is significantly inhibited.

\section{Reference}

[1] K. Fu , L. Ma, H.F. Li, Research of Water Hammer Based on Flowmaster, J. Refrigeration and Air Conditioning. 29 (2015) 597-600.

[2] X.X. Mei, L.D. Tang, Y. Tang, Application of FLOWMASTER to Hydraulic Balance in Air Bonditioning Bhilled Water System, J. HV\&AC. 44 (2014) 98-101.

[3] Z.Y. Jiao, H. Liu, The Applycation of Fluid Simulation Software Flowmaster in Cooling Water Pump Configration Analysis, J. Metallurgical Power. 4 (2009) 89-93. 